\title{
Body Lateropulsion With Involvement of the Medial Longitudinal Fasciculus Due to a Rostral Pontine Tegmentum Infarction
}

\author{
Hiromasa Tsuda $^{\mathrm{a}, \mathrm{b}}$, Motohiro Fujiwara ${ }^{\mathrm{a}}$, Toshio Kaneda ${ }^{\mathrm{a}}$
}

\begin{abstract}
Patient 1 developed leftward body lateropulsion (BL), right internuclear ophthalmoplegia, and hypalgesia and thermohypoesthesia in the territory of the left trigeminal nerve due to an infarction in the right rostral ponine tegmentum. Patient 2 developed rightward $\mathrm{BL}$ and left non-paralytic pontine exotropia without alternating exotropia due to an infarction in the left rostral pontine tegmentum. Although impairment of the ascending graviceptive pathway (GP) causes BL, its precise location remains uncertain. Neurologic findings in our patients suggest that GP may run in the vicinity of the medial longitudinal fasciculus, ventral trigemino-thalamic tract and paramedian pontine reticular formation in the rostral pons.
\end{abstract}

Keywords: Ascending graviceptive pathway; Internuclear ophthalmoplegia; Non-paralytic pontine exotropia; Paramedian pontine reticular formation; Skew deviation; Ventral trigemino-thalamic tract

\section{Introduction}

Body lateropulsion (BL) is defined as irresistible falling to one side without motor weakness [1,2]. Although the ascending graviceptive pathway (GP) from the vestibular nuclei to the Cajal interstitial nucleus crosses the midline in the caudal pons, just above the level of the vestibular nuclei

Manuscript accepted for publication December 9, 2013

${ }^{a}$ Department of Neurology, Tokyo Metropolitan Health and Medical Corporation Toshima Hospital, 33-1, Sakaecho, Itabashi-ku, 173-0015 Tokyo, Japan

${ }^{\mathrm{b}}$ Corresponding author: Hiromasa Tsuda, Department of

Neurology, Tokyo Metropolitan Health and Medical Corporation

Toshima Hospital, 33-1, Sakaecho, Itabashi-ku, 173-0015 Tokyo,

Japan. Email: hiromasa_tsuda@tokyo-hmt.jp

doi: http://dx.doi.org/10.14740/jmc1635w
[1], its precise location remains uncertain in the rostral pons. Here, we describe two cases of BL with involvement of the medial longitudinal fasciculus (MLF) due to rostral pontine tegmentum infarction. The neurologic symptoms in these two cases are very useful for determining of the location of GP.

\section{Case Report}

\section{Patient 1}

An 80-year-old man without contributory medical history suddenly presented with leftward BL, right-sided internuclear ophthalmoplegia (INO) and left-sided hypalgesia and thermohypoesthesia in the territory of the maxillary as well as mandibular nerve in August 2012. There were no other neurologic abnormalities. Laboratory examination was within normal ranges. Chest X-ray finding was normal. Electrocardiogram demonstrated no abnormalities. Diffusionweighted magnetic resonance (MR) imaging demonstrated a small infarction in the rostral ponine tegmentum on the right side (Fig. 1A). Cranial MR angiography finding was normal. Two days of intravenous argatroban hydrate therapy $60 \mathrm{mg} /$ day was initiated. Thereafter, 5 days of argatroban hydrate $20 \mathrm{mg}$ /day underwent, the patient became gradually asymptomatic.

\section{Patient 2}

A 56-year-old woman with essential hypertension abruptly presented with rightward $\mathrm{BL}$, left-sided non-paralytic pontine exotropia (NPPE) without alternating exotropia, and comitant skew deviation with left-sided hypertropia (Fig. 2) in August 2013. There were no other neurologic abnormalities. Laboratory examination was within normal ranges. Chest $\mathrm{X}$-ray finding was normal. Electrocardiogram demonstrated no abnormalities. Diffusion-weighted MR imaging demonstrated a small infarction in the rostral ponine tegmentum on the left side (Fig. 1B). Cranial MR angiography finding was normal. Under ozagrel sodium $160 \mathrm{mg}$ /day, the patient became gradually asymptomatic within 7 days. 


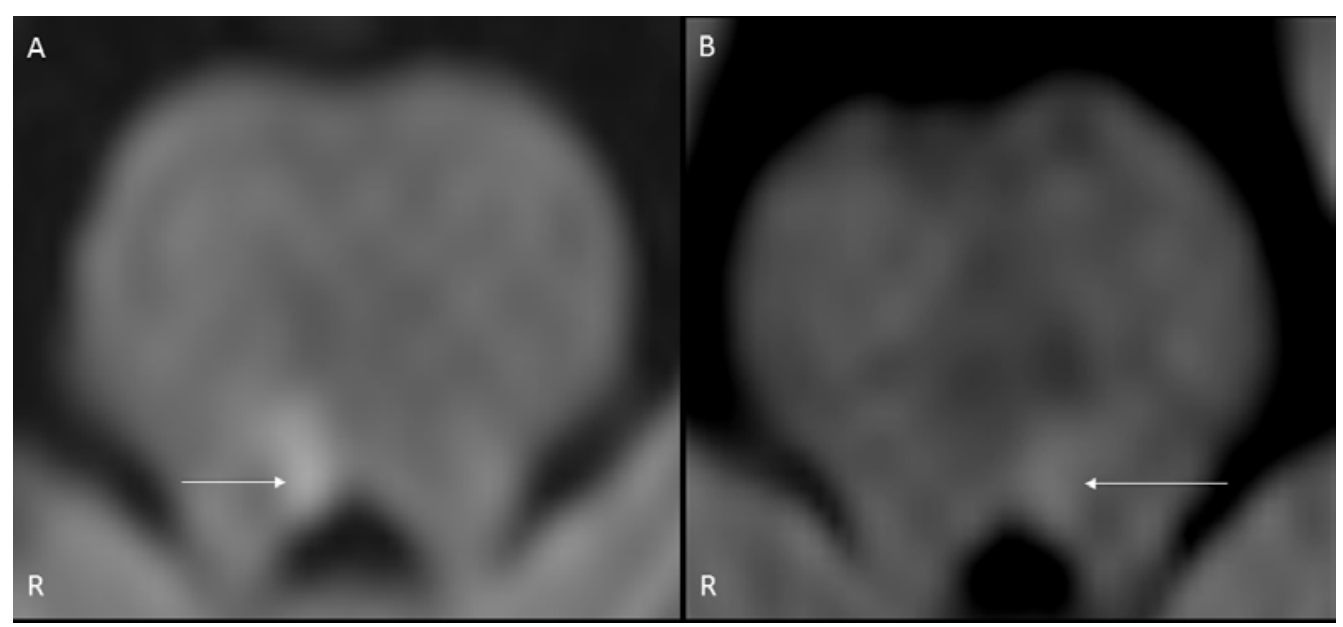

Figure 1. Cranial diffusion-weighted magnetic resonance imaging (A: patient 1, B: patient 2). A: A small infarction was demonstrated in the rostral pontine tegmentum on the right side (arrow). B: A small infarction was demonstrated in the left rostral pontine tegmentum on the left side (arrow).

\section{Discussion}

Yi et al [1] reported five cases of BL due to rostral pontine tegmentum infarction, and the direction of BL was to the side opposite the lesions in all cases. Moreover, because unilateral INO was observed in four of these five cases, the authors speculated that GP might run in the vicinity of the MLF [1]. Tsuda et al [2] noted a case of BL with hypalgesia and thermophypoesthesia in the territory of all divisions of the trigeminal nerve caused by a rostral pontine tegmentum infarction, and stated that GP might run in the vicinity of the ventral trigemino-thalamic tract in the rostral pons. In our present patient 1 , in addition to leftward BL, right-sided INO and left-sided hypalgesia and thermohypoesthesia in the territory of the maxillary as well as mandibular nerve was caused by infarction in the right rostral pontine tegmen- tum. This neurologic finding denoted that GP might run in between MLF and the ventral trigemino-thalamic tract in the rostral pons.

NPPE is defined as unilateral INO with exotropia of the contralateral eye $[3,4]$. NPPE without alternating exotropia is caused by complete involvement of the MLF as well as incomplete damage to the paramedian pontine reticular formation (PPRF) [3]. On the other hand, comitant skew deviation may be induced by abnormalities in the midbrain, pons, medulla oblongata, cerebellum and thalamus, and is considered as a poorly localizing sign of posterior fossa dysfunction [5]. However, because comitant skew deviation with INO frequently develops, it is considered that damage to the MLF may cause comitant skew deviation [6]. In our present patient 2 , in addition to rightward BL, left-sided NPPE without alternating exotropia, and comitant skew deviation were ob-
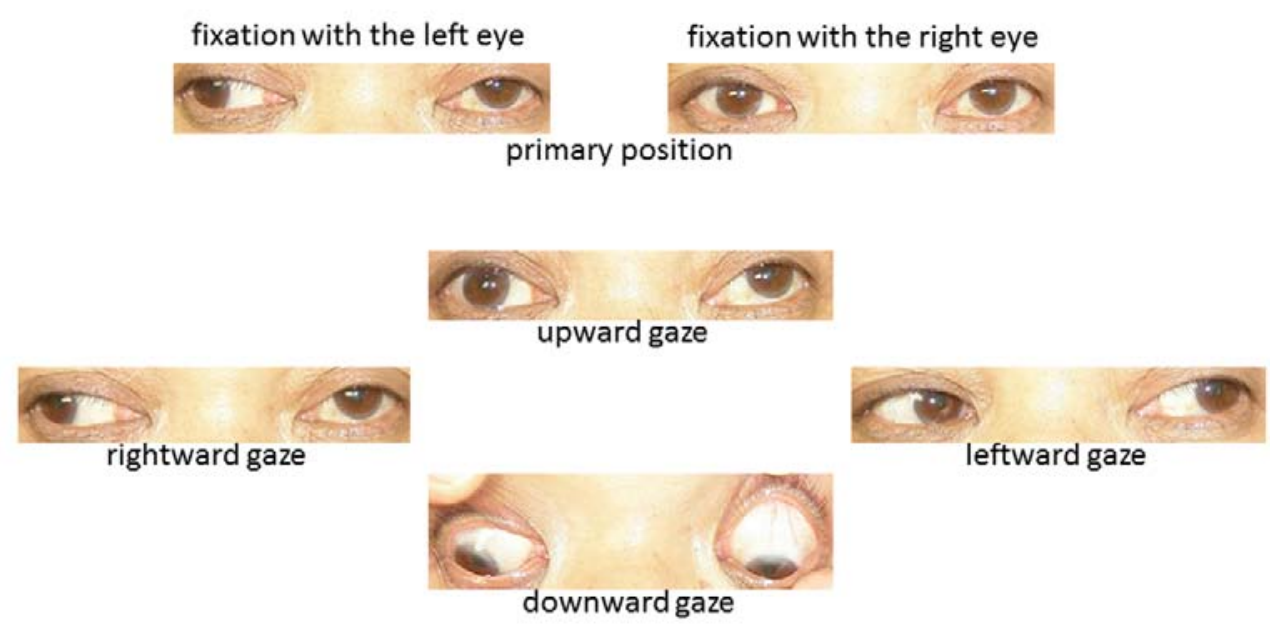

Figure 2. Ocular motor finding in patient 2. Left-sided non-paralytic pontine exotropia without alternating exotropia as well as comitant skew deviation with left-sided hypertropia was observed. 
served. These neuro-ophthalmologic findings suggested that GP might run in the vicinity of MLF as well as PPRF in the rostral pons.

In conclusion, we emphasize that GP may run in between the MLF and ventral trigemino-thalamic tract as well as in the vicinity of PPRF in the rostral pons, based on the neurologic symptoms of our two patients.

\section{Grant Support}

None.

\section{References}

1. Yi HA, Kim HA, Lee H, Baloh RW. Body lateropulsion as an isolated or predominant symptom of a pontine infarction. J Neurol Neurosurg Psychiatry.
2007;78(4):372-374.

2. Tsuda H, Koh S, Tanaka K. Body lateropulsion with hypalgesia and thermophypoesthesia in the territory of all divisions of the trigeminal nerve caused by a pontine infarction. Jpn J Stroke. 2013;35(3):213-215.

3. Takamatsu K, Ohta T. A study of eight patients with non-paralytic pontine exotropia. Rinsho Shinkeigaku. 1995;35(10):1110-1113.

4. Komiyama A, Takamatsu K, Johkura K, Hasegawa O, Fukutake T, Hirayama K. Internuclear ophthalmoplegia and contralateral exotropia. Nonparalytic pontine exotropia and WEBINO syndrome. Neuroophthalmology. 1998;19(1):33-44.

5. Keane JR. Ocular skew deviation. Analysis of 100 cases. Arch Neurol. 1975;32(3):185-190.

6. Zwergal A, Cnyrim C, Arbusow V, Glaser M, Fesl G, Brandt T, Strupp M. Unilateral INO is associated with ocular tilt reaction in pontomesencephalic lesions: INO plus. Neurology. 2008;71(8):590-593. 